\title{
Research Paper \\ The Prevalence and Etiology of Ankle Injury in Professional Karate Players in Iran
}

\author{
*Hamidreza Naserpour ${ }^{1}\left(\mathbb{C}\right.$, Mona Mirjani $^{2}$ (]
}

1. Department of Biomechanics and Sport Injuries, Faculty of Physical Education and Sports Sciences, Kharazmi University, Tehran, Iran. 2. Department of Sport Injuries and Corrective Exersice, Faculty of Physical Education \& Sport Sciences, University of Tehran, Tehran, Iran

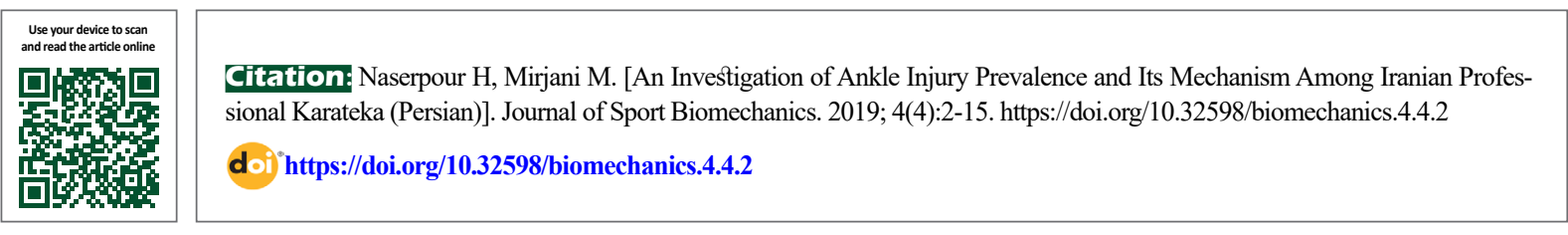

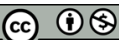

Article Info:

Received: 10 Nov 2018 Accepted: 13 Jan 2019 Available Online: 01 Mar 2019

Keywords:

Prevalence, Etiology, ankle injury, Foot and ankle ability measure, Karate

\section{ABSTRACT}

Objective: The aim of this study was to investigate the etiology of ankle injury and its prevalence among the Iranian professional karate players.

Methods: Participants were 390 karate kumite players (Mean \pm SD of age $=24.5 \pm 2.8$ years; Mean \pm SD of weight $=63.5 \pm 1.8 \mathrm{~kg}$ ) attendant in the competitions for the national team selection. Foot and Ankle Ability Measure (FAAM) questionnaire was used for assessing their information of ankle injury such as history, etiology, received treatments, and its effects on their daily living performance and sports activities. Descriptive statistics (Mean $\pm S D$ ) were used to describe the data, and Pearson correlation test was used to evaluate the relationship between daily living and sports scores obtained from FAAM and those self-reported scores at a significance level of $\mathrm{P} \leq 0.05$.

Results: Results was reported that $86 \%$ of subjects had a history of ankle injury, and most of them occurred during the competition. The most important reason for ankle injury was sudden rotation of the foot and kicking the opponent. In most cases, subjects had self-treatment and did not visit a physician. Correlation coefficients of daily living and sports scores obtained from FAAM with athletes' self-reported scores were 0.588 and $0.742(\mathrm{P}=0.000)$, respectively.

Conclusion: Regarding the prevalence of ankle injury among karate players, it is recommended that specific proprioceptive and strengthening programs be put on the agenda for coaches and athletes to reduce the risk factors of injury. Due to the athletes' lack of follow-up for treatment and rehabilitation in most injuries, there is a possibility of re-injury to the ankle; therefore, the recovery process should be performed after injury under the supervision of a physician and returning to training should be done after proper interval.

\section{Extended Abstract}

\section{Introduction}

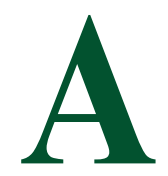

thletes are always exposed to the potential harm that can be reduced if being aware of its cause, treatment, and appropriate preventive measures [9]. Low risks and minor injuries associated with karate are commonly believed by athletes $[10,11]$. Previous research has reported less injury in karate, compared to other sports [10]. Accordingly, most injuries in karate include blows, sprains, and minor injuries. Serious injuries, including fractures and sprains, are more common in sports, such as football, wrestling, weightlifting, skiing, running, taekwondo, judo, volleyball, and basketball [9, 10, 12]. It is essential for coaches, athletes, and physicians to understand the relevant etiology, prevention, and treatment; this might help to recognize which body parts are most affected $[12,13]$. Numerous studies have suggested that

\section{* Corresponding Author:}

\section{Hamidreza Naserpour, PhD. Student.}

Address: Department of Biomechanics and Sport Injuries, Faculty of Physical Education and Sports Sciences, Kharazmi University, Tehran, Iran. Tel: +98 (936) 6798689

E-Mail: hamidreza.naserpour@gmail.com 
the ankle joint is most vulnerable to injury, compared to other body joints [17-20]; approximately $2 \%$ of all injuries are related to this part of the lower limb [19]. To increase safety in sport, a mechanism and its associated factors should be identified, followed by preventive measures to reduce injury [9]. Injury prevention strategies can change the injury profile in different sports through effective interventions. Injury profile widely varies in different sports; therefore, preventive measures must be appropriate to the sport to prevent injury [24]. The current study investigated the prevalence and etiology of ankle injury in professional karate players in Iran.

\section{Participants and Methods}

This was a descriptive retrospective study. In coordination with the Karate Federation of the Islamic Republic of Iran, 390 karate players in fighting style (kumite) participants in the national team selection were recruited based on a convenience sampling technique. Data collection tools were: 1. A demographic data form surveying height, weight, age, and Body Mass Index (BMI); 2. Sports Information Questionnaire, surveying sports experience, the number of training sessions, the number of days and hours of training, the duration of training, the type and duration of warm-up; 3. Ankle Injury History Questionnaire, surveying injury history, injured leg (dominant and non-dominant), the causes of injury, the time of injury, and the type of treatment and aftercare treatments; 4. Foot and Ankle Ability Measure (FAAM), i.e. a self-report instrument. It is designed to measure musculoskeletal disorders of the lower extremities as well as physical function in patients with ankle instability and injuries [25].

\section{Results}

Table 1 provides information on the history of ankle injury in the study participants. The Mean \pm SD scores of the two subscales of daily living and sports obtained from FAAM were $86.88 \pm 11.31$ and $84.29 \pm 15.54$, respectively. The study subjects' self-reported Mean \pm SD scores on the subscale of daily living and sports were $87.21 \pm 13.11$ and $86.59 \pm 14.42$, respectively. Furthermore, correlation coefficients of the two subscales with self-reported scores were $0.588(\mathrm{P}=0.000)$ and $0.742(\mathrm{P}=0.000)$, respectively.

\section{Discussion}

The present study investigated the prevalence and etiology of ankle injury among professional karate players in Iran. In this regard, we identified the factors affecting the injury and the extent of ankle joint motor self-awareness in the study subjects. By identifying the causes and factors involved in the ankle injury, then taking preventive measures to reduce the extent of the injury, a comprehensive program could be formulated to reduce its financial and mental burdens. In total, $86 \%$ (335) of the study athletes reported a history of an ankle injury, with $>50 \%$ of these injuries occurring over the past year. Ankle injuries in both feet, dominant leg and non-dominant leg were the most frequent injuries, respectively. Of these, $46 \%$ reported that they sometimes had impaired sports performance and $25 \%$ reported that ankle injuries had no negative impact on their performance. This finding is consistent with those of Rahimi et al. [27], Sahebozamani et al. [28] and Del Vecchio et al. [29]. Given the widespread prevalence of injury in athletes, special strengthening and proprioceptive training programs should be considered for coaches and athletes to reduce the extent of such injuries.

\section{Conclusion}

In total, $86 \%$ of karate players reported ankle sprains and injuries; the highest injury occurred during the competition. The most common causes of ankle injury were the sudden rotation of the foot and kicking the opponent.

Table 1. The history of ankle injury in the study participants

\begin{tabular}{|c|c|c|}
\hline & & $\%$ \\
\hline \multirow{3}{*}{ History of ankle sprain } & Lifetime & 86.9 \\
\hline & Past year & 44.4 \\
\hline & Past 6 months & 32.3 \\
\hline \multirow{4}{*}{ Frequency of ankle sprain in the dominant leg } & Once & 32.3 \\
\hline & $2-4$ & 37.9 \\
\hline & $>5$ & 7.9 \\
\hline & No injury & 21.8 \\
\hline
\end{tabular}




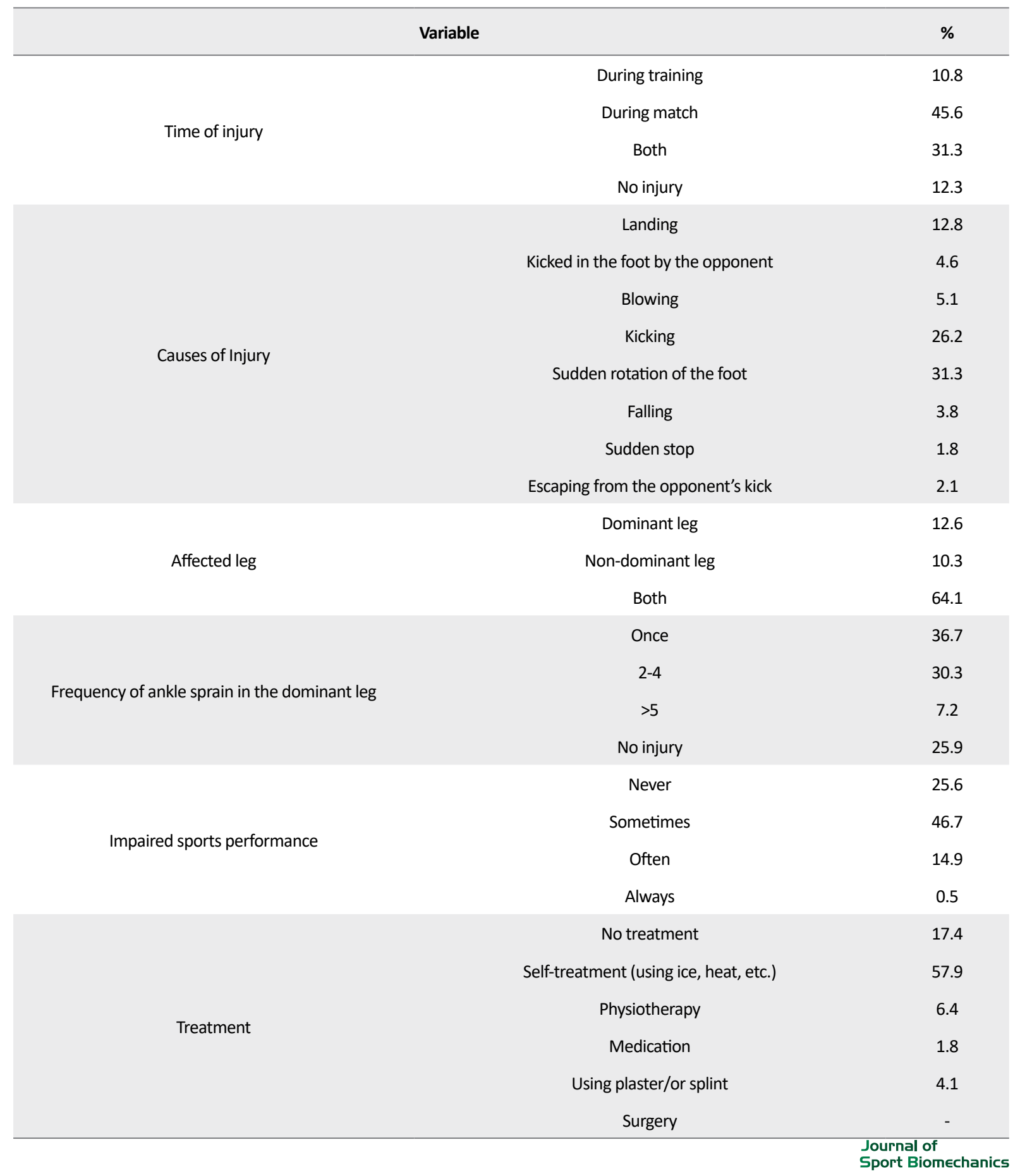

Given the prevalence of injuries, it is recommended that proprioceptive and strengthening programs be considered for coaches and athletes to reduce injury risk in this population. Due to the athletes' lack of follow-up for treatment and rehabilitation in most injuries, there is a possibility of re-injury to the ankle; therefore, the recovery process should be performed after injury under the supervision of a physician. Besides, returning to training should occur after a proper interval.

\section{Ethical Considerations}

\section{Compliance with ethical guidelines}

Prior to the study, participants were informed of the study objectives and methods and an informed consent was obtained from them. They were assured of the confidentiality of their information. 


\section{Funding}

This research did not receive any financial support from funding agencies in the public, commercial, or not-forprofit organizations.

\section{Authors' contributions}

Conceptualization, methodology, validation, formal analysis, investigation, resources, witing-original draft preparation, writing-review \& editing, visualization, supervision, project administration: All authors.

\section{Conflicts of interest}

The authors declare no conflict of interest.

\section{Acknowledgements}

The authors would like to thank President and Vice President of the Karate Federation of Iran and all participants and those involved in national team competitions for their valuable cooperation. 
This Page Intentionally Left Blank 


\title{
شيوع و بررسى مكانيسم آسيب ممج ياى كاراتهكاران حرفاى ايران
}

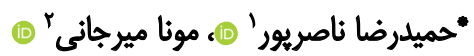

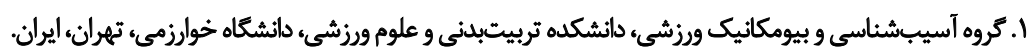

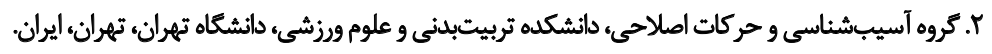

\begin{abstract}
حكيد
هدف: هدف از اين مطالعه، شيوع و بررسى اثواع مكانيسم آسيب ميج باى كاراتهكاران حرفهاى ايران بان بودان.

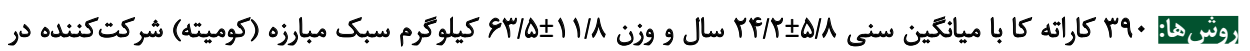

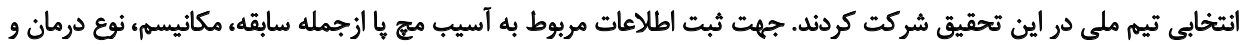

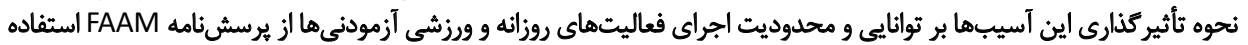

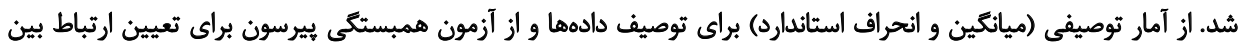

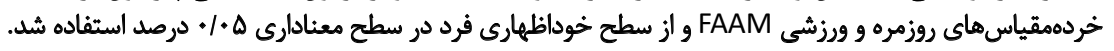

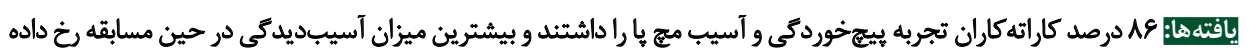

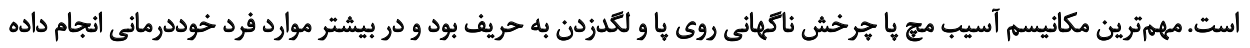

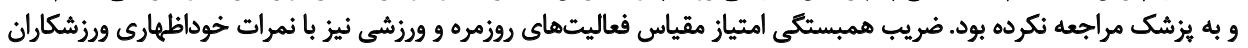

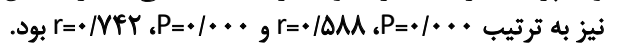

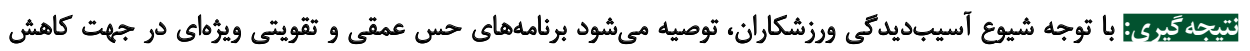

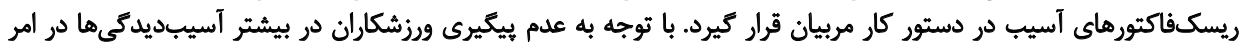

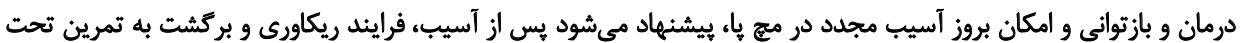
نظر يزشك انجام كيرد.
\end{abstract}

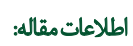

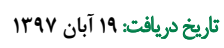

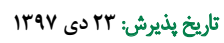

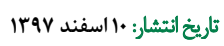

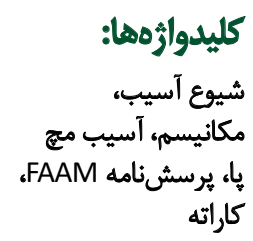

شركت مردم در فعاليتهاى ورزشى بخش خاصى از زند ازتى إنى

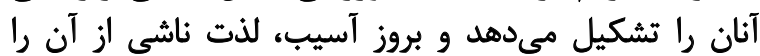

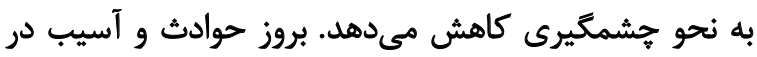

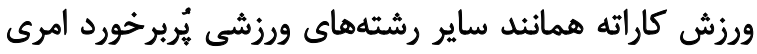

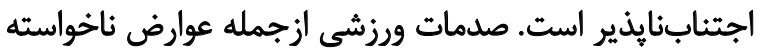

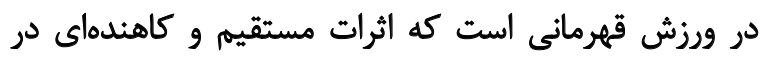

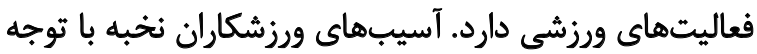

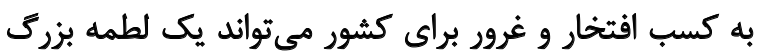
اجتماعى براى آن كشور به حساب بيايد.

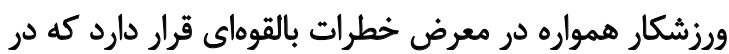

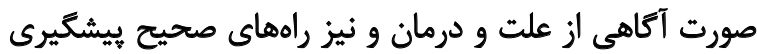

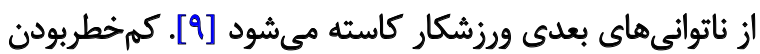

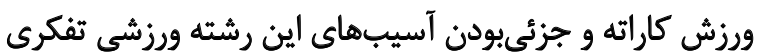

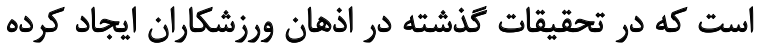

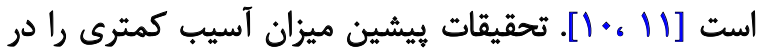

ورزش هاى رزمى از كشورهاى جين، كره و زاين نشئت كرفته و

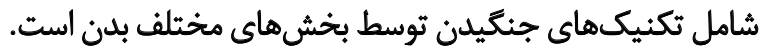

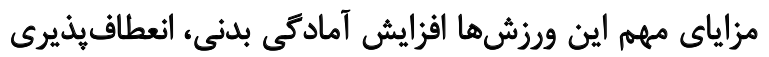

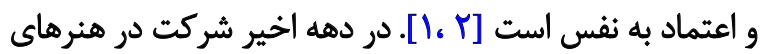

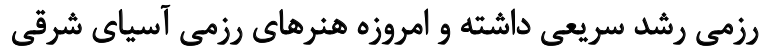

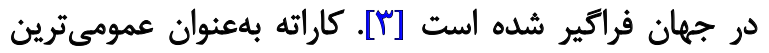

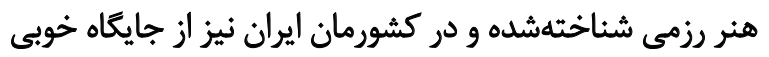

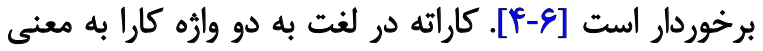

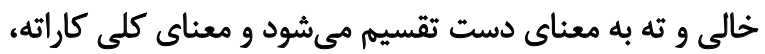

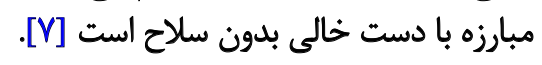
مسابقات كاراته به دو صورت كاتا (فرم) و كميته (مبارزه) انجام

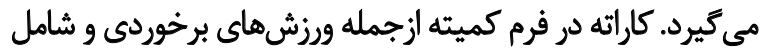

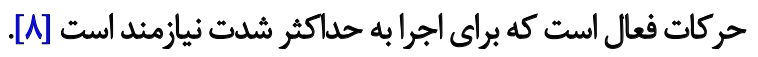

$$
\begin{aligned}
& \text { : نويسئده مسئول: } \\
& \text { حميدرضا ثاصريور }
\end{aligned}
$$

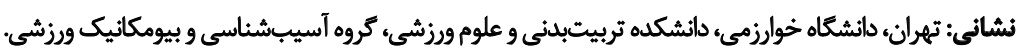

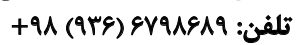




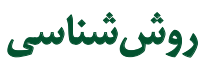

تحقيق حاضر از نوع توصيفى و كذنشتهنكر است. با توجه

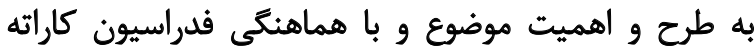

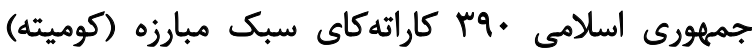

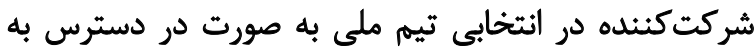

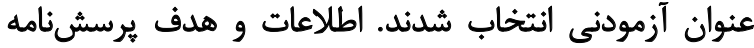

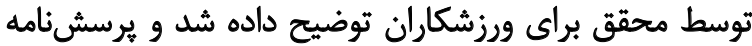

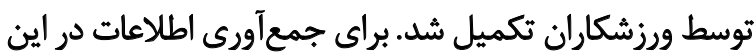

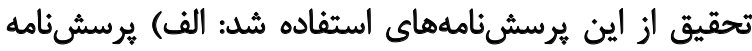

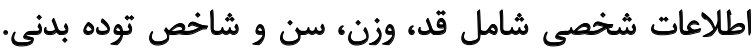

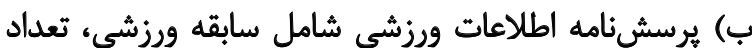

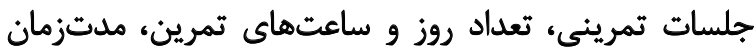

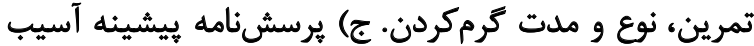

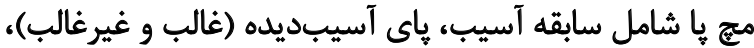

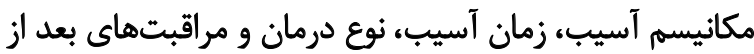

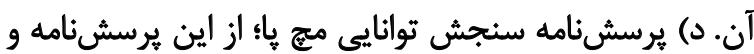

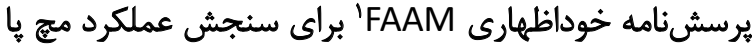
در فعاليتهاى روزانه و ورزش استفاده شداي

مقياس سنجش توانايى يا و ميج با براي اندازمكيرى اختلالات

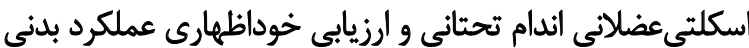

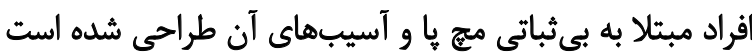

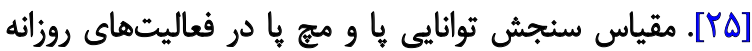

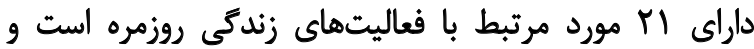

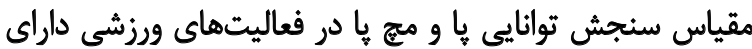

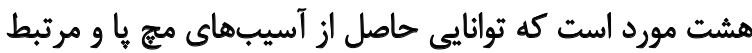

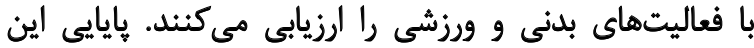

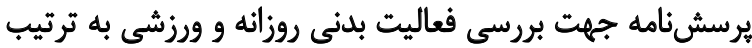

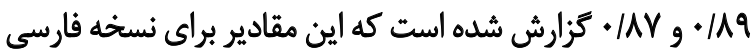

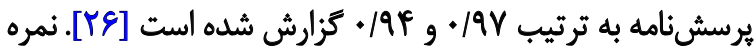

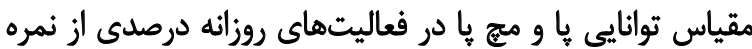

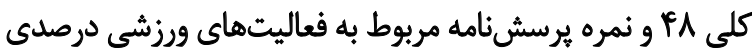

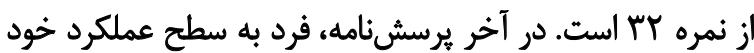

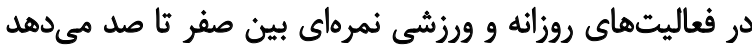

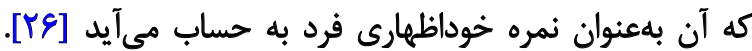

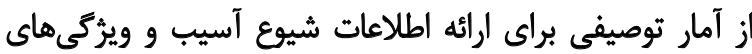

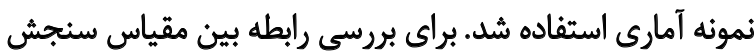

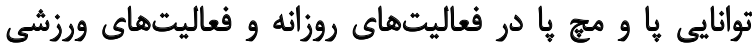

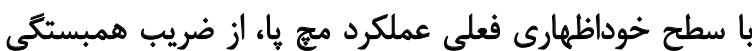

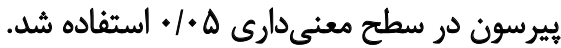

كاراته در مقايسه با ساير رشتهاي ورزشى كزارش كردهاند [ • 1]. بر اين اساس بيشترين آسيبها در كارئه

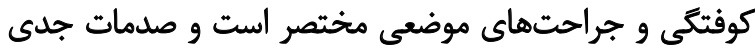

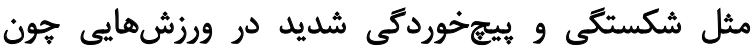

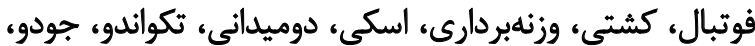

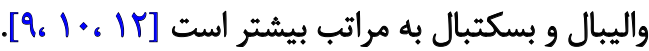
اين نكته كه كدام قسمتهاي بدن بيشتر دهار آسيب

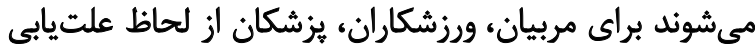

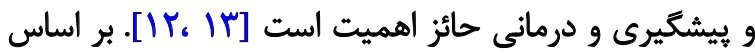

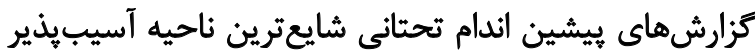

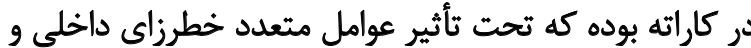

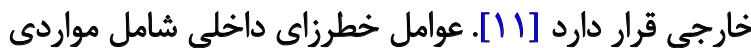

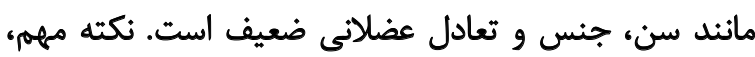

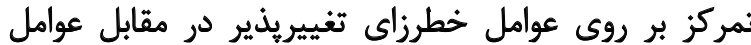

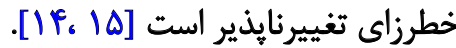

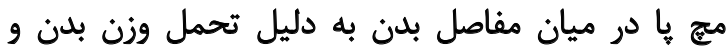

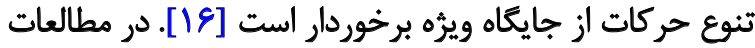

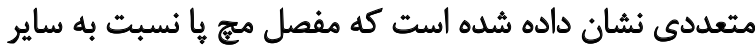

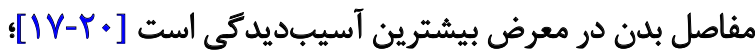

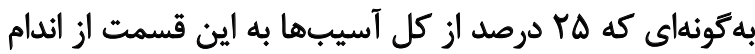

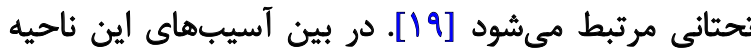

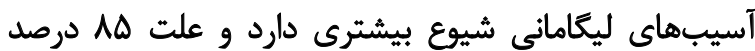

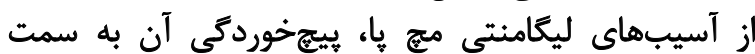

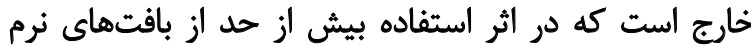

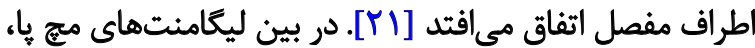

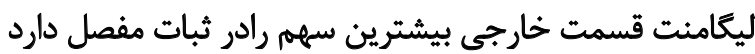

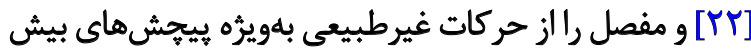

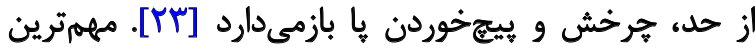

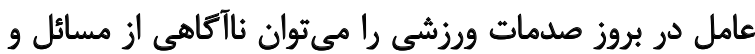

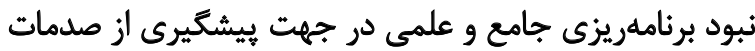

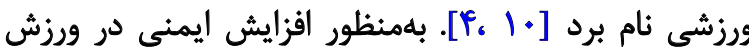

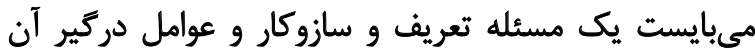

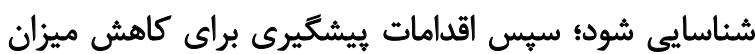

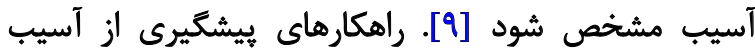

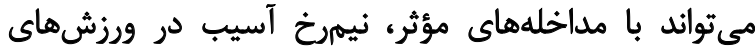

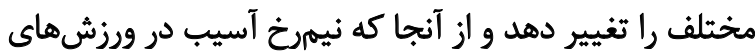
مختلف به صورت كسترده با هم تفاوت دات دارد؛ بنابراين براي آنداي

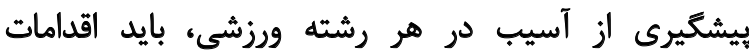

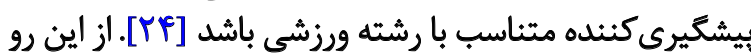

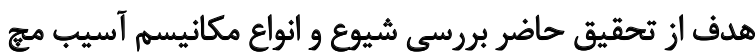

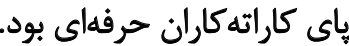


جدول ا. مشخصات فردى آزمودنىها

\begin{tabular}{|c|c|c|c|c|}
\hline شاخص توده بدنى & 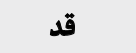 & 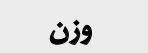 & سن & متغير \\
\hline$r / \varepsilon \pm \Delta V / \lambda$ & $1 \gamma \pm \pm 1 \cdot 10$ & $g \Psi / 1 \backslash \pm \Delta / \lambda$ & $r r / \Delta \pm r / \lambda$ & انحراف استاندارد土ميانكين \\
\hline
\end{tabular}

مجله بيومكانيك ورنش

برنامه مشخص و جامع در جهت كاهش هزينههاى مالى و

$$
\text { روحى تدوين كرد. }
$$

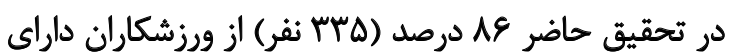

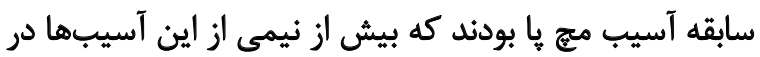

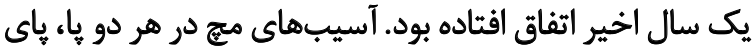

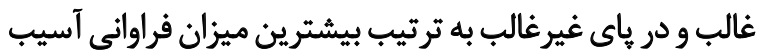

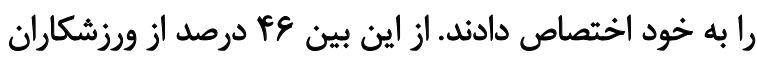

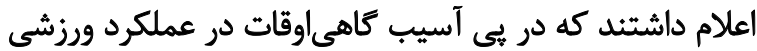

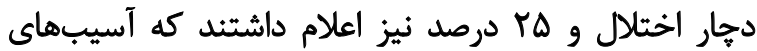

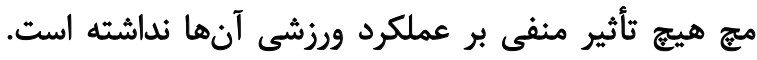

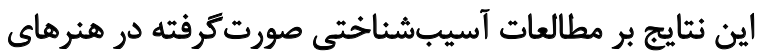

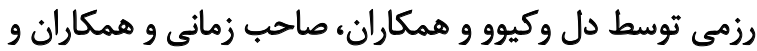

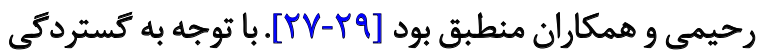

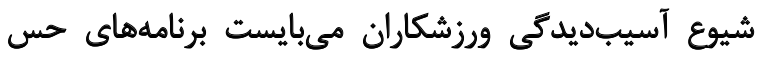

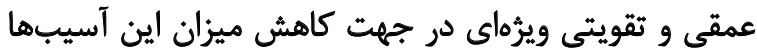
در دستور كار مربيان و ورزشكاران قرار كيردي

على ترغم زمان حضور بيشتر ورزشكاران در برنامهانهاي

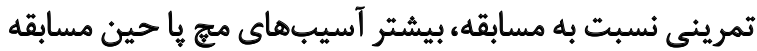

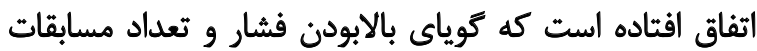

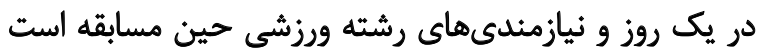

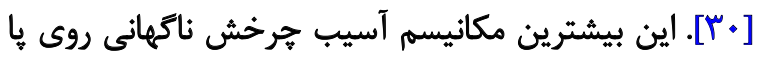

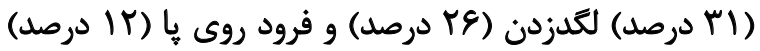

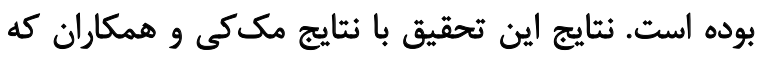

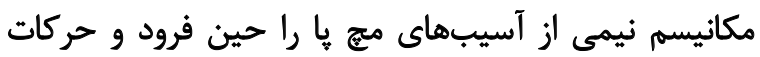

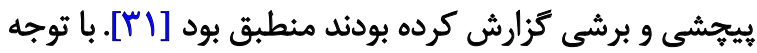

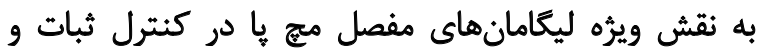

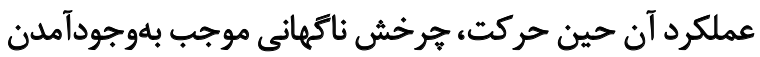

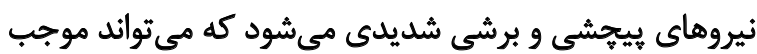

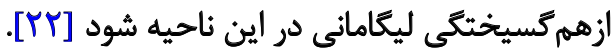

در حدود Y I درصد از ورزشكاران آسيبديده نياز به مداواي

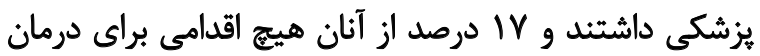

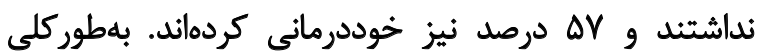

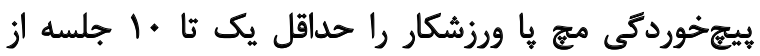

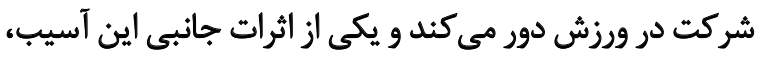

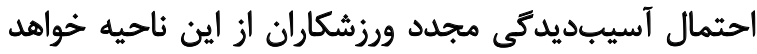

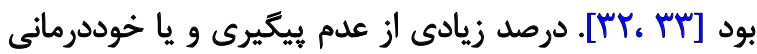

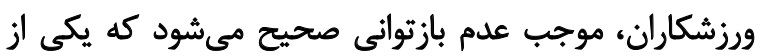

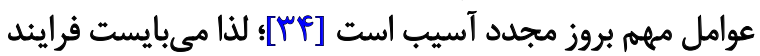

مشخصات جمعيتشناختى آزمودنىها در جدول شماره الرائه

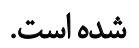

در جدول شماره r نيز اطلاعات ورزشى آزمودنىها بهات إنهات

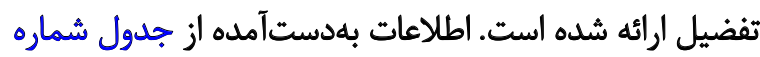

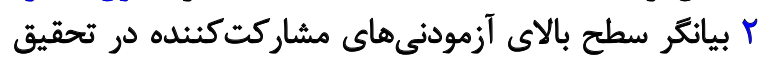

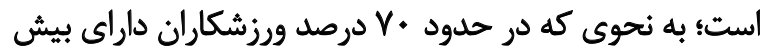

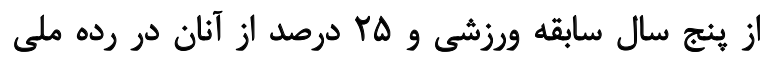
فعاليت مي كردند.

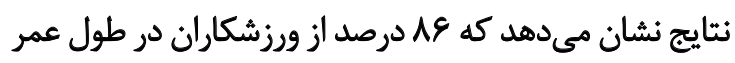

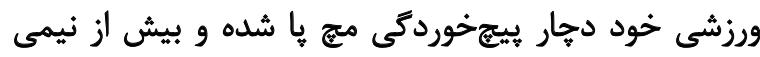

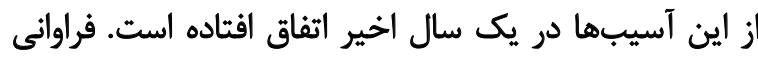

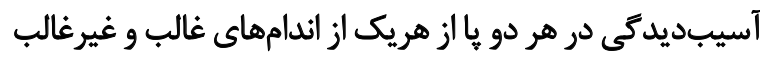

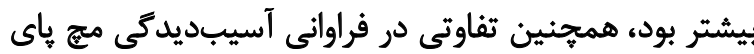

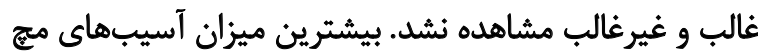

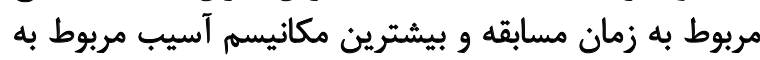

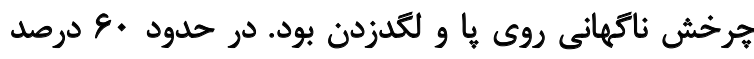

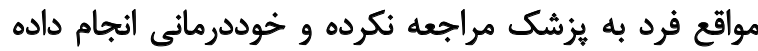

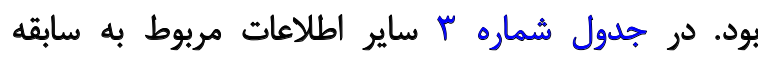

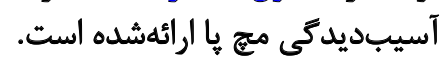

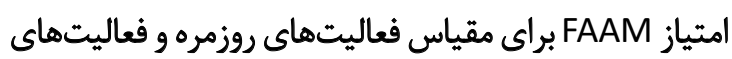

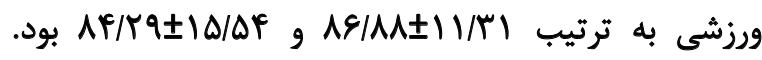

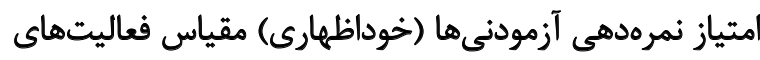

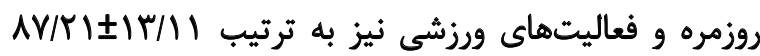

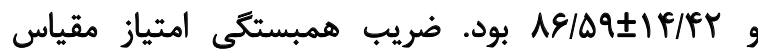

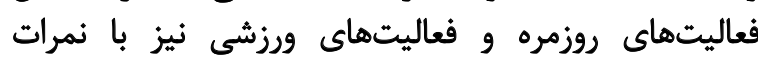

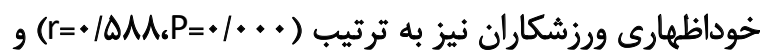
بود.

بحث

هدف از تحقيق حاضر بررسى شيوع و مكانيسم آسيب

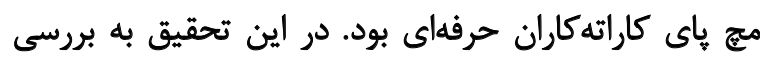

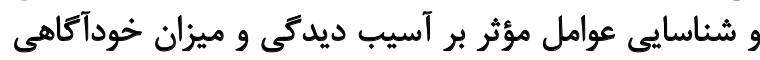

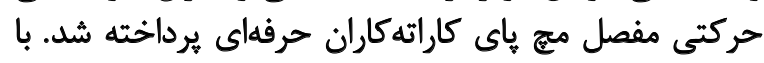

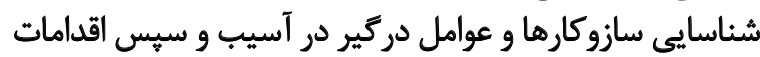

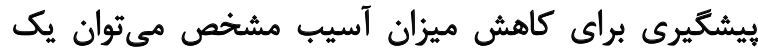


جدول ץ.اطلاعات ورزشى آزمودنىها

\begin{tabular}{|c|c|c|}
\hline$x$ & & \\
\hline$r \Delta / 1$ & 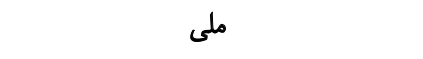 & \multirow{3}{*}{ سطح ورزشى } \\
\hline$v e / 9$ & باشكاهى & \\
\hline- & داششكاهى و آموزشكاهى & \\
\hline$\cdot / \Lambda$ & ( - ا-ז روز & \multirow{4}{*}{ تعداد روزهاى تمرين در هفته } \\
\hline 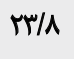 & r & \\
\hline 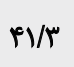 & 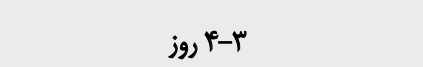 & \\
\hline$m / 1$ & $\Delta$ & \\
\hline$r / T$ & | اجلسه & \multirow{8}{*}{ هلتزمان كرمكردن در هر جلسه } \\
\hline$m \varphi / q$ & 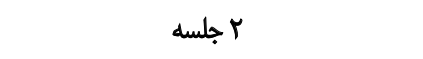 & \\
\hline$r V / r$ & r إجلسه & \\
\hline$\cdot N$ & 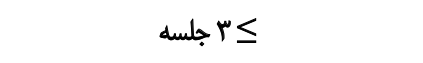 & \\
\hline $1 \Delta / \%$ & 1ه دقيقه & \\
\hline$v \xi / 8$ & | إ-بr دقيقه & \\
\hline$V / \Delta$ & |r-r|r|r دقيقه & \\
\hline .10 & 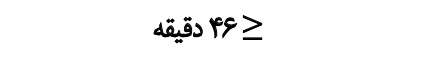 & \\
\hline$\cdot 11$ & 1> ساعت & \multirow{4}{*}{ مدتزمان هر جلسه تمرينى } \\
\hline gV/P & 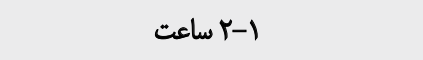 & \\
\hline rNT & r-r باعت & \\
\hline$r / q$ & Ir & \\
\hline $1 / r$ & (ll $r \geq$ & \multirow{3}{*}{ سابقه تمرين به صورث جلى } \\
\hline$r q$ & ب- به سال & \\
\hline ga/ & S ال & \\
\hline $\mathrm{r} / \mathrm{A}$ & | l ب جلسه & \multirow{4}{*}{ تعداد جلسات تمرين در هفته } \\
\hline rq & 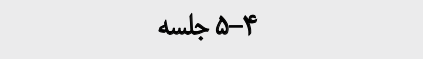 & \\
\hline $19 / \Delta$ & هـ- - جلسه & \\
\hline $19 / \mathrm{V}$ & > & \\
\hline$V / r$ & حركات كثشى & \multirow{6}{*}{ نوع كرمكردن } \\
\hline Na & دويدن & \\
\hline IV/T & تمرينات تخصصى ورزشى & \\
\hline $19 / \mathrm{V}$ & حركات كششى و دويدن & \\
\hline NA & حركات كششى و تمرينات تخصصى & \\
\hline$r / /$ & حركات كششى، دويدن و تمرينات تخصصى & \\
\hline
\end{tabular}

مجله بيومكانيكوزنش 
خودآكاهى متوسطى از وضعيت مفصل ميج ياى خود حين

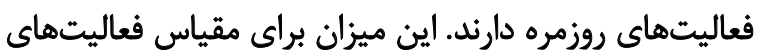

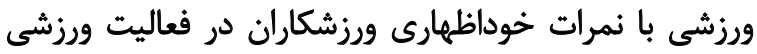

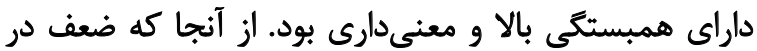

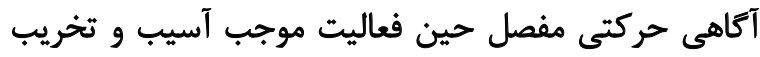

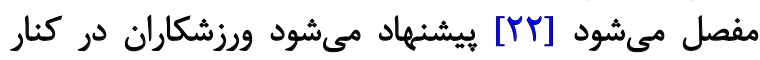

ريكاورى يس از آسيب تحت نظر يزشك و با رعايت فاصله زمانى مناسب جهت شروع مجدد برنامه تمرينى لحاظ شود. نتايج آزمون آمارى همبستكى معنى دار و متوسطى را براى

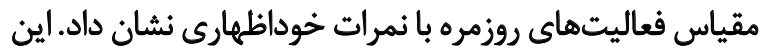
نتايج نشان داد كه ورزشكاران شركت بكنينده در تحقيق دارئ

جدول ץ.اطلاعات مربوط به سابقه بيجخوردكى مـج ڤا

\begin{tabular}{|c|c|c|}
\hline درصد & & \\
\hline$N / 9$ & در طول عمر & \multirow{3}{*}{ سابقه ييجنوردمى } \\
\hline$\mu \varepsilon / \varepsilon$ & يك سال اخير & \\
\hline$\pi / \%$ & شش ماه اخير & \\
\hline$\pi / \%$ & البار & \multirow{4}{*}{ عداد دفعات ييجخوردكى ياى غالب 1} \\
\hline$r / q$ & 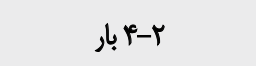 & \\
\hline V/a & بيشتر از ينج بار & \\
\hline$r / \Lambda$ & بلدون آسيب & \\
\hline reN & إبار & \multirow{4}{*}{ عداد دفعات ييجخوردمى ياى غالب r } \\
\hline$r+/ r$ & 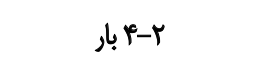 & \\
\hline$v / r$ & بيشتر از ينج بار & \\
\hline$r \Delta / q$ & بلون أسيب & \\
\hline $1+11$ & تمرين & \multirow{4}{*}{ زمان وقوع آسيب } \\
\hline PA/S & مسابقه & \\
\hline$r / r$ & هر دو ل & \\
\hline$\pi / r$ & بلدون آسيب & \\
\hline $\mid r / A$ & فرود & \multirow{8}{*}{ مكائيسم أسيب } \\
\hline$r / 8$ & لكششن با توسط حريف & \\
\hline$\Delta / 1$ & اصابت ضربه & \\
\hline re/r & ل لكدزدن & \\
\hline m/r & هرخش ناكمانى روى يا & \\
\hline$r / A$ & افتادن & \\
\hline $1 / 4$ & توقف ناكهانى & \\
\hline$r /$ & فرار از ضربه حريف & \\
\hline $\mid r / 8$ & بالى غالب & \multirow{3}{*}{ باى دجار ييجخوردىى } \\
\hline $1+/ r$ & باى غيرغالب & \\
\hline$\$ * 1$ & هر دو با & \\
\hline TA/Q & هركز & \multirow{4}{*}{ اختلال در عملكرد ورزشى } \\
\hline $\operatorname{ren}$ & كاهمىاوقات & \\
\hline $18 / 9$ & اغلب|وقات & \\
\hline.$/ \Delta$ & هميشه & \\
\hline
\end{tabular}




\begin{tabular}{|c|c|c|}
\hline درصد & مثتير & \\
\hline $\mid V / f$ & هيج درمانى نكردم & \\
\hline$\Delta V / q$ & (يخخ و خوما و غيرمان) & \\
\hline $8 / 4$ & فيزيوترايى & درمان آسيب \\
\hline$v / A$ & درمان يزشكى (دارو) & \\
\hline$r /$ & كّي يا آتل & \\
\hline- & جراحى & \\
\hline
\end{tabular}

مجله بيومكانيكورنش

تمرينات تخصصى خود به تمرينات حس عمقى و حركتى نيز مشاركت نويسند

بيردازند.

مفهومسازى، روششناسى، اعتبارسنجى، تحليل، تحقيق و

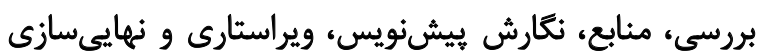

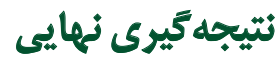

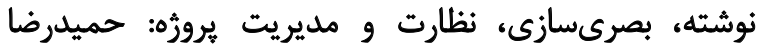

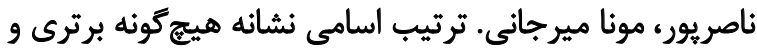

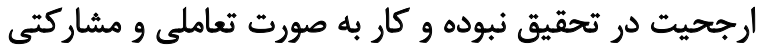

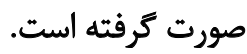

تعارض منافع

بنــا بــه اظهـار نويسـندكًان ايسن مقالـهـ تعــارض منافـع

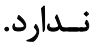

$$
\text { تشكر و قدردانى }
$$

در هايان از مساعدتهاى رياست و نايبرئيس فدراسيون

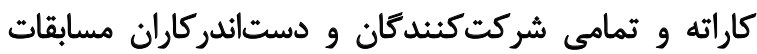

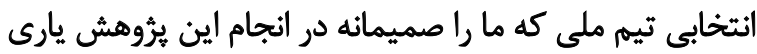

$$
\text { رساندند، تشكر و قدرداني مي كنيم. }
$$

نتايج اين مطالعه نشان داد ع1 دروصد كاراته كاران حاضر در

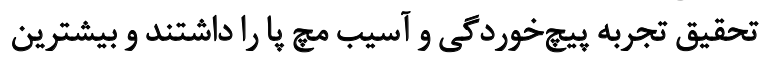

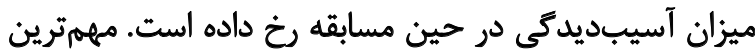

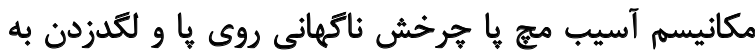

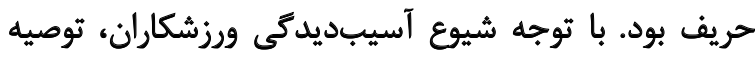

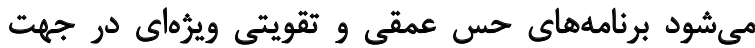

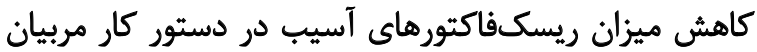

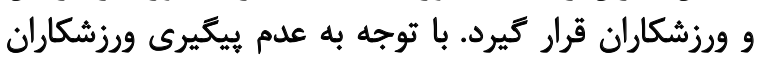

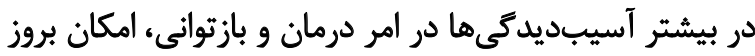

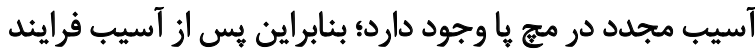

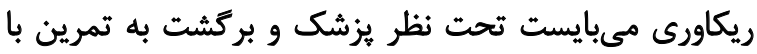
رعايت فاصله زمانى مناسب انجام كيرد.

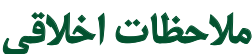

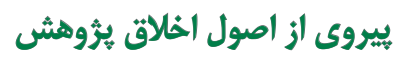

تمام مراحل براى آزمودنى ها به صورت شفاهى توسط محقق

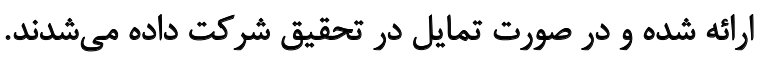

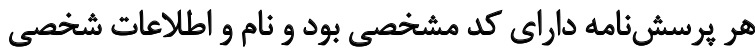

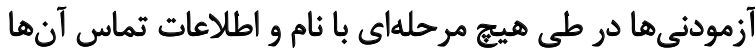

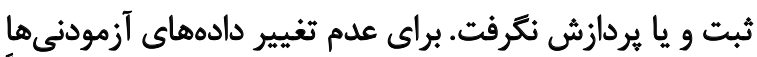

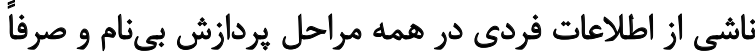
با كد برسش إمامه صورت كرفته است.

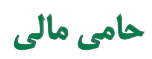

مقاله فوق با هزينه شخصى و تحت نظر هيج نهاد و يا اركان

$$
\text { دولتى و غير دولتى انجام نكرفته است }
$$




\section{References}

[1] Kazemi M, Shearer H, Choung YS. Pre-competition habits and injuries in Taekwondo athletes. BMC Musculoskeletal Disorders. 2005; 6:26. [DOI:10.1186/1471-2474-6-26] [PMID] [PMCID]

[2] Viswanath Y, Rogers I. A non-contact complete knee dislocation with popliteal artery disruption, a rare martial arts injury. Postgraduate Medical Journal. 1999; 75(887):552-4. [DOI:10.1136/ pgmj.75.887.552] [PMID] [PMCID]

[3] Pieter W. Martial arts injuries. Medicine and Sport Science. 2005; 48:59-73. [DOI:10.1159/000084283] [PMID]

[4] Critchley GR, Mannion S, Meredith C. Injury rates in Shotokan karate. British Journal of Sports Medicine. 1999; 33(3):174-7. [DOI:10.1136/bjsm.33.3.174] [PMID] [PMCID]

[5] Kazemi M, Waalen J, Morgan C, White AR. A profile of Olympic taekwondo competitors. Journal of Sports Science \& Medicine. 2006; 5(CSSI):114-21. [PMID] [PMCID]

[6] Zetaruk MN, Violan MA, Zarakowski D, Micheli LJ. Karate injuries in children and adolescents. Accident Analysis \& Prevention. 2000; 32(3):421-5. [DOI:10.1016/s0001-4575(99)00120-7] [PMID]

[7] Yard EE, Knox CL, Smith GA, Comstock RD. Pediatric martial arts injuries presenting to Emergency Departments, United States 1990-2003. Journal of Science and Medicine in Sport. 2007 10(4):219-26. [DOI:10.1016/j.jsams.2006.06.016] [PMID]

[8] Beneke R, Beyer T, Jachner C, Erasmus J, Hütler M. Energetics of karate kumite. European Journal of Applied Physiology. 2004; 92(4-5):518-23. [DOI:10.1007/s00421-004-1073-x] [PMID]

[9] Burke DR. Treating martial arts injuries [K. Sharifi Azar, Persian trans.]. Tehran: Elmoharekat; 1998.

[10] Arriaza R, Leyes M. Injury profile in competitive karate: Prospec tive analysis of three consecutive World Karate Championships. Knee Surgery, Sports Traumatology, Arthroscopy. 2005; 13(7):6037. [DOI:10.1007/s00167-004-0593-6] [PMID]

[11] Sharma L, Song J, Felson DT, Cahue S, Shamiyeh E, Dunlop DD. The role of knee alignment in disease progression and functional decline in knee osteoarthritis. JAMA. 2001; 286(2):188-95 [DOI:10.1001/jama.286.2.188] [PMID]

[12] Destombe C, Lejeune L, Guillodo Y, Roudaut A, Jousse S Devauchelle $V$, et al. Incidence and nature of karate injuries. Joint Bone Spine. 2006; 73(2):182-8. [DOI:10.1016/j.jbspin.2005.02.003] [PMID]

[13] Dadgar H, Sahebozamani M, Noorai T, Sharifian E. [The relationship between $\mathrm{Q}$-angle and non-contact injuries of lower extremity in male karate players (Persian)]. Journal of Sport Medicine. 2011; 1(3):83-97.

[14] Bahr R, Krosshaug T. Understanding injury mechanisms: A key component of preventing injuries in sport. British Journal of Sports Medicine. 2005; 39(6):324-9. [DOI:10.1136/bjsm.2005.018341] [PMID] [PMCID]

[15] McCrory P. Clinical guide to sports injuries. British Journal of Sports Medicine. 2006; 40(6):561. [DOI:10.1136/ bjsm.2005.022939] [PMCID]

[16] Naserpour HR, Sadeghi H. [The effect of short-term use of cold spray on strength and ankle joint position sense in professional wrestlers (Persian)]. Journal of Sport Biomechanics. 2017; $3(2): 43-50$.
[17] Lee HM, Cheng CK, Liau JJ. Correlation between proprioception muscle strength, knee laxity, and dynamic standing balance in patients with chronic anterior cruciate ligament deficiency. The Knee. 2009; 16(5):387-91. [DOI:10.1016/j.knee.2009.01.006] [PMID]

[18] Beynnon BD, Renström PA, Alosa DM, Baumhauer JF, Vacek PM. Ankle ligament injury risk factors: A prospective study of college athletes. Journal of Orthopaedic Research. 2001; 19(2):213-20. [DOI:10.1016/S0736-0266(00)90004-4]

[19] McGuine TA, Greene J, Best T, Leverson G. Balance as a predictor of ankle injuries in high school basketball players. Clinical Journa of Sport Medicine. 2000; 10(4):239-44. [DOI:10.1097/00042752 200010000-00003] [PMID]

[20] de Noronha M, Refshauge KM, Herbert RD, Kilbreath SL, Hertel J. Do voluntary strength, proprioception, range of motion, or postural sway predict occurrence of lateral ankle sprain? British Journal of Sports Medicine. 2006; 40(10):824-8. [DOI:10.1136/ bjsm.2006.029645] [PMID] [PMCID]

[21] Ozmun JC, Thieme HA, Ingersoll CD, Knight KL. Cooling does not affect knee proprioception. Journal of Athletic Training. 1996; 31(1):8-11. [PMID] [PMCID]

[22] Hrysomallis C, Goodman C. A review of resistance exercise and posture realignment. Journal of Strength and Conditioning Research. 2001; 15(3):385-90. [DOI:10.1519/00124278-20010800000022] [PMID]

[23] Leanderson J, Eriksson E, Nilsson C, Wykman A. Proprioception in classical ballet dancers: A prospective study of the influence of an ankle sprain on proprioception in the ankle joint. The American Journal of Sports Medicine. 1996; 24(3):370-4. [DOI:10.1177/036 354659602400320] [PMID]

[24] Livingston LA, Spaulding SJ. OPTOTRAK measurement of the quadriceps angle using standardized foot positions. Journal of Athletic Training. 2002; 37(3):252-5. [PMID] [PMCID]

[25] Martin RL, Irrgang JJ, Burdett RG, Conti SF, Van Swearingen JM. Evidence of validity for the Foot and Ankle Ability Measure (FAAM). Foot \& Ankle International. 2005; 26(11):968-83. [DOI:10 1177/107110070502601113] [PMID]

[26] Mazaheri M, Salavati M, Negahban H, Sohani SM, Taghizadeh F, Feizi $A$, et al. Reliability and validity of the Persian version of Foot and Ankle Ability Measure (FAAM) to measure functional limitations in patients with foot and ankle disorders. Osteoarthritis and Cartilage. 2010; 18(6):755-9. [DOI:10.1016/j.joca.2010.03.006] [PMID]

[27] Rahimi M, Halabchi F, Ghasemi GA, Zolaktaf V. [Prevalence of karate injuries in professional karate kai in Isfahan (Persian)]. Annals of Military and Health Sciences Research. 2009; 7(3):201-7.

[28] Sahebozamani M, Beyranvand R. [A review of injury assessment in Iranian martial artists: Systematic review (Persian)]. Scientific Journal of Rehabilitation Medicine. 2016; 5(2):235-48

[29] Del Vecchio FB, Farias CB, de Leon RC, Rocha ACCA, Galliano LM, Coswig VS. Injuries in martial arts and combat sports: Prevalence, characteristics and mechanisms. Science \& Sports. 2018; 33(3):158-63. [DOI:10.1016/j.scispo.2018.02.003]

[30] Venter S, Janse Van Rensburg DC, Fletcher L, Grant CC. The prevalence, risk factors predicting injury and the severity of injuries sustained during competition in professional mixed martial arts in Africa. South African Journal of Sports Medicine. 2017; 29(1):1-5. [DOI:10.17159/2078-516x/2017/v29i0a1471] 
[31] McKay GD, Goldie PA, Payne WR, Oakes BW. Ankle injuries in basketball: Injury rate and risk factors. British Journal of Sports Medicine. 2001; 35(2):103-8. [DOI:10.1136/bjsm.35.2.103] [PMID] [PMCID]

[32] Kofotolis N, Kellis E. Ankle sprain injuries: A 2-year prospective cohort study in female Greek professional basketball players. Journal of Athletic Training. 2007; 42(3):388-94. [PMID] [PMCID]

[33] Bahr R, Engebretsen L. Handbook of sports medicine and science, sports injury prevention. Hoboken: Wiley; 2009. [DOI:10.1002/9781444303612]

[34] Khayambashi Kh, Ghoddosi N, Falah AR. [Prediction of ankle and knee injuries with static leg balance test (Persian)]. Journal of Sport Medicine Review. 2017; 8(20):17-36. 
This Page Intentionally Left Blank 